\title{
Strong coupling probe for the Kardar-Parisi-Zhang equation
}

\author{
T.J. Newman ${ }^{(1)}$ 月 and Harald Kallabis ${ }^{(2)}$ \\ (1) Institut für Theoretische Physik, Universität zu Köln, D-50937 Köln, Germany \\ (2) Höchstleistungsrechenzentrum, Forschungszentrum Jülich, 52425 Jülich, Germany
}

(July 24, 2021)

\begin{abstract}
We present an exact solution of the deterministic Kardar-Parisi-Zhang (KPZ) equation under the influence of a local driving force $f$. For substrate dimension $d \leq 2$ we recover the well-known result that for arbitrarily small $f>0$, the interface develops a non-zero velocity $v(f)$. Novel behaviour is found in the strong-coupling regime for $d>2$, in which $f$ must exceed a critical force $f_{c}$ in order to drive the interface with constant velocity. We find $v(f) \sim\left(f-f_{c}\right)^{\alpha(d)}$ for $f \searrow f_{c}$. In particular, the exponent $\alpha(d)=2 /(d-2)$ for $2<d<4$, but saturates at $\alpha(d)=1$ for $d>4$, indicating that for this simple problem, there exists a finite upper critical dimension $d_{u}=4$. For $d>2$ the surface distortion caused by the applied force scales logarithmically with distance within a critical radius $R_{c} \sim\left(f-f_{c}\right)^{-\nu(d)}$, where $\nu(d)=\alpha(d) / 2$. Connections between these results, and the critical properties of the weak/strong-coupling transition in the noisy KPZ equation are pursued.
\end{abstract}

PACS numbers: 03.65, 05.40.+j, 68.35.R

Short title: Strong coupling probe for the KPZ equation 


\section{INTRODUCTION}

This article is concerned with a problem which arises in the study of the Kardar-ParisiZhang (KPZ) equation [1] of interface growth [2] [3]. Namely, we consider the deterministic version of the KPZ equation under the influence of a local, constant force. Although this problem is in principle rather simple, a detailed investigation has not been carried out to our knowledge. The results, although interesting in their own right, assume greater significance when related to the weak/strong-coupling transition in the noisy KPZ equation. Before entering into further details of this problem, we shall give a brief overview of the key properties of the KPZ equation. In, by now, standard notation this equation for the evolution of the interface height $h(\mathbf{x}, t)$ (above a $d$-dimensional substrate) takes the form

$$
\partial_{t} h=\nu \nabla^{2} h+(\lambda / 2)(\nabla h)^{2}+\eta
$$

where $\eta(\mathbf{x}, t)$ is a white noise, generally taken to be gaussian distributed. The Cole-Hopf transformation $h(\mathbf{x}, t)=(2 \nu / \lambda) \ln (w(\mathbf{x}, t))$ yields a linear equation for $w$ albeit with multiplicative noise:

$$
\partial_{t} w=\nu \nabla^{2} w+(\lambda / 2 \nu) w \eta
$$

This equation may be re-expressed by means of the Feynman-Kac formula, such that $w$ represents the restricted partition function of a directed polymer in the presence of quenched random defects. Naturally, the above equation may also be interpreted as an imaginary-time Schrödinger equation for a quantum mechanical (QM) particle in a time-dependent random potential $(-\eta)$.

It is well-known [面] that $d=2$ plays the role of a lower critical dimension. For $d \leq 2$, the interface will be asymptotically (i.e. for large times) rough for arbitrarily small $\lambda$. For $d>2$ there exists a transition between asymptotically smooth and rough phases, depending on the value of $\lambda$. To access the rough (strong-coupling) phase, $\lambda$ must exceed a critical value $\lambda_{c}$. Amongst the most important questions in this field are: i) what are the scaling exponents 
associated with the strong-coupling phase; ii) does there exist an upper critical dimension $d_{u}$, above which these exponents take on mean-field values; iii) what are the critical properties of the weak/strong-coupling transition? There are no answers to the first question, bar predictions from extensive numerical work (except for the case $d=1$ where exact analytic results are available [2]). As regards the second question, the community seems split between beliefs in $d_{u}=4$ and $d_{u}=\infty$. The former belief is based primarily on the predictions of various self-consistent 'mode-coupling' theories (see for example [4]), whilst the latter belief arises from numerical evidence for $d$-dependent strong-coupling exponents [3] (for all accessible $d \lesssim 7$ ). With regard to the final question, a recent two-loop renormalization group calculation [5] (formulated as an $\epsilon$-expansion, with $\epsilon=d-2$ ) predicts that at the transition, the roughness exponent $\chi=0+O\left(\epsilon^{3}\right)$ (to be interpreted as logarithmic roughness), whilst the correlation length exponent has the form $\tilde{\nu}=(1 / \epsilon)+O\left(\epsilon^{3}\right)$. For the one-loop versions of these results, see Ref. [6].

We now concentrate on a much simpler problem. As mentioned before, we retain the essential non-linearity of the KPZ equation, but replace the noise $\eta$ by a local applied force: $\eta(\mathbf{x}, t)=f_{0} \delta^{d}(\mathbf{x})$. In directed polymer language this corresponds to replacing the bulk disorder by a single constant energy columnar defect. In terms of the QM analogue, we have replaced the random potential by a delta-function potential with amplitude $\left(-f_{0}\right)$. There exists a well-known result [7], which is most easily stated in QM language: for $d \leq 2$ the particle will have a bound state for arbitrarily small $f_{0}>0$, whereas for $d>2$ there only exists a bound state for $f_{0}>f_{0, c}>0$. In terms of the interface, these results imply that for $d \leq 2$ an arbitrarily small force is sufficient to induce a non-zero velocity to the surface, whereas for $d>2$ the driving force must exceed a critical value in order to achieve this. The identification of a lower critical dimension (along with the properties of the system for $d \leq 2$ ) may be obtained from a perturbative analysis (in terms of $\lambda$, or equivalently $f_{0}$ ).

In order to explore the properties of the 'binding/unbinding' transition for $d>2$, a non-perturbative analysis is required - such an analysis constitutes the body of the present work. The main results which arise are the following (which we shall describe in terms of 
the interface formulation). Whereas for $d<2$ an arbitrarily small driving force will induce a velocity $v\left(f_{0}\right)$ to the interface, for $d>2$ the applied force must exceed a critical value in order to achieve this. The dependence of the velocity on the excess force $\delta f_{0} \equiv f_{0}-f_{0, c}$ is found to be $v \sim\left(\delta f_{0}\right)^{\alpha(d)}$ where $\alpha(d)=2 /(d-2)$ for $2<d<4$, but saturates at $\alpha(d)=1$ for $d>4$. For $d<2$ the applied force will create a non-linear distortion of the surface over

a critical radius $R_{c} \sim f_{0}^{-1 /(2-d)}$, but the amplitude of the distortion is of order unity. For $d>2$ the distortion scales logarithmically with distance and exists within a critical radius $R_{c} \sim\left(\delta f_{0}\right)^{-\nu(d)}$, where $\nu(d)=\alpha(d) / 2$. Therefore, for $d>2$ the distortion is a macroscopic rupture of the surface with a logarithmic profile (by 'rupture' we mean a gross distortion of the interface, leaving the continuous properties of the surface in tact). We shall leave the interpretation of these results for the final sections of the paper.

The outline of the paper is as follows. In the next section we shall give a precise definition of the model to be solved, along with some simple steps which lead to an accessible strong-coupling (i.e. non-perturbative) analysis of the model. In section 3, we proceed with this analysis and derive the velocity-force characteristics. The spatial profiles of the surface distortion for various dimensions are evaluated in section 4 . Connections between the properties of this simple model, and the weak/strong-coupling transition in the noisy KPZ model are pursued in section 5. We end the paper with our conclusions, and a description of possible extensions to the present work.

\section{DEFINITION AND REFORMULATION OF THE MODEL}

Replacing the stochastic source of the noisy KPZ equation with a local applied force leads us to consider the equation

$$
\partial_{t} h=\nu \nabla^{2} h+(\lambda / 2)(\nabla h)^{2}+f_{0} \delta^{d}(\mathbf{x})
$$

As for the noisy problem, we may apply the transformation $h(\mathbf{x}, t)=(2 \nu / \lambda) \ln (w(\mathbf{x}, t))$ which yields the linear equation 


$$
\partial_{t} w=\nu \nabla^{2} w+\left(\lambda f_{0} / 2 \nu\right) w \delta^{d}(\mathbf{x})
$$

Since we are primarily interested in the interface application of this problem, we choose the initial condition $w(\mathbf{x}, 0)=1$, which corresponds to an initially flat interface $(h=0)$. [The natural initial condition for the directed polymer application is $w(\mathbf{x}, 0)=\delta^{d}(\mathbf{x})$. We shall not consider this problem here.]

We may formally integrate Eq.(田) using the Green function of the diffusion equation, $g(\mathbf{x}, t)=(4 \pi \nu t)^{-d / 2} \exp \left(-x^{2} / 4 \nu t\right)$, leading to

$$
w(\mathbf{x}, t)=1+\left(\lambda f_{0} / 2 \nu\right) \int_{0}^{t} d t^{\prime} g\left(\mathbf{x}, t-t^{\prime}\right) w\left(\mathbf{0}, t^{\prime}\right) .
$$

Therefore, knowledge of the function $w(\mathbf{0}, t)$ is sufficient to determine $w$ throughout all space. For convenience we shall define $\phi(t) \equiv w(\mathbf{0}, t)$. Setting $\mathbf{x}=\mathbf{0}$ in the above equation leads to a Volterra integral equation for $\phi$ :

$$
\phi(t)=1+f \int_{0}^{t} d t^{\prime} \sigma\left(t-t^{\prime}\right) \phi\left(t^{\prime}\right)
$$

where $\sigma(t)=t^{-d / 2}$ and we have defined an effective force $f \equiv\left(\lambda f_{0} / 2 \nu\right)(4 \pi \nu)^{-d / 2}$. [Such a Volterra equation may also be derived for the more complicated situation of a time-dependent force $f(t)$. The case where $f(t)$ is a stochastic variable is of particular interest in the directed polymer picture [8].]

Clearly this equation is ill-defined for $d>2$, since the kernel is non-integrable over the range $(0, t)$. To regularize the equation we have many possibilities. The most convenient is to smear the delta-function force over a small region of size $l^{d}$ with a gaussian envelope, i.e. we replace $f_{0} \delta^{d}(\mathbf{x})$ by $f_{0}\left(\pi l^{2}\right)^{-d / 2} \exp \left(-x^{2} / l^{2}\right)$. The length scale $l$ constitutes the smallest physical scale in the problem. Assuming that $w(\mathbf{x}, t)$ varies slowly over the core region $|\mathbf{x}|<l$ allows us to rederive the above Volterra equation, where now $\sigma(t)=\left(t+l^{2}\right)^{-d / 2}$ (after rescaling $l \rightarrow l=l /(4 \nu))$.

Laplace transforming Eq.(可) and using the convolution theorem yields an explicit solution for this Laplace transform $\mathcal{L}[\phi(t)]$. Inverting the transform gives the result 


$$
\phi(t)=\frac{1}{2 \pi i} \int_{\gamma} \frac{d s}{s} \frac{e^{s t / l^{2}}}{\left[1-l^{2-d} f D(s)\right]}
$$

where $\gamma$ is the usual integration contour along the line $s=c+i y$ where $c$ is a real constant chosen to lie to the right of any singularities in the complex plane. The function $D(s)$ is given by

$$
D(s)=\int_{0}^{\infty} d y \frac{e^{-s y}}{(1+y)^{d / 2}}
$$

(which may be expressed in terms of the incomplete Gamma function [9]).

We are now in a position to obtain all physical properties of the model from the analysis of Eq.(7). We stress once again that this analysis is non-perturbative in $f_{0}$ (or equivalently $\lambda$ ). All the results for $d \leq 2$ may be obtained by perturbation theory (although one must evaluate all orders), whereas none of the results concerning the binding/unbinding transition may be obtained by such a technique for $d>2$.

In the next section we shall concentrate on evaluating the asymptotic (large time) form of $\phi(t)$ which will enable us to determine whether the interface has a non-zero velocity. The spatial profile of the interface distortion requires the evaluation of the convolution of the diffusion equation Green function with the function $\phi(t)$ (as illustrated in Eq. (5)) - this analysis is described in section 4 .

\section{VELOCITY-FORCE CHARACTERISTICS}

The singularities in the integrand of Eq.(7) consist of a branch cut along the negative real axis, and (depending on the value of $f$ ) a pole in the right-half plane (RHP). The large-time behaviour of $\phi(t)$ will be dominated by the contribution from the pole (if it exists). One may easily convince oneself that the existence of this pole is required for the interface at the forcing site to have a non-zero velocity, i.e. for $H(t) \sim v t($ where $H(t) \equiv(\lambda / 2 \nu) h(\mathbf{0}, t)=\ln \phi(t)$.)

By studying the small-s behaviour of $D(s)$ one may show the following properties of the integrand in Eq.(7). For $d<2$ there always exists a pole in the RHP for $f>0$, which for 
$f \searrow 0$ is situated at $s_{p}=f^{2 /(2-d)}$. For $d>2$ the pole will only exist in the RHP for $f>$ $f_{c}=l^{d-2}(d-2) / 2$. For $f \searrow f_{c}$, the position of the pole is given by $s_{p}=A(d)\left(f-f_{c}\right)^{2 /(d-2)}$ for $2<d<4$ (where $\mathrm{A}(\mathrm{d}$ ) is a complicated function of $d$ ), whereas for $d>4$ one has $s_{p}=[(d-4) /(d-2)]\left(f-f_{c}\right)$. This abrupt change of behaviour for $s_{p}$ at $d=4$ will have interesting consequences.

Evaluating the contribution to $\phi(t)$ from the pole leads us to the following results for the velocity-force characteristics: i) for $d<2$ one has for $f \searrow 0$ the relation $v(f) \sim f^{2 /(2-d)}$; ii) for $d>2$, the velocity is zero unless $f$ exceeds $f_{c}$ - one then has for $f \searrow f_{c}$ the relation $v(f) \sim(\delta f)^{\alpha(d)}$ where $\delta f \equiv f-f_{c}$ and

$$
\alpha(d)= \begin{cases}\frac{2}{(d-2)} & 2<d<4 \\ 1 & d>4,\end{cases}
$$

which signals the existence of an upper critical dimension $d_{u}=4$.

In the absence of a pole in the RHP, the asymptotics of $\phi(t)$ arise from the contribution from the branch cut. The results for this case (along with the $v(f)$ characteristics at the critical dimensions 2 and 4) are displayed for completeness in Table I.

\section{SPATIAL PROFILES}

In this section we wish to evaluate the spatial profile of the interface for $f>f_{c}$. We define the positive quantity $\Delta H(\mathbf{x}, t) \equiv(\lambda / 2 \nu)(h(\mathbf{0}, t)-h(\mathbf{x}, t))$. Making use of the ColeHopf transformation, along with Eq. (5), we have (after rescaling $\mathbf{x} \rightarrow \mathbf{x}=\mathbf{x} /(4 \nu)$ )

$$
\Delta H=-\ln \left\{\frac{1}{\phi(t)}+\frac{f}{\phi(t)} \int_{0}^{t} d t^{\prime} g\left(\mathbf{x}, t^{\prime}+l^{2}\right) \phi\left(t-t^{\prime}\right)\right\} .
$$

For $f>f_{c}$ and $v t \gg 1$, we have from the previous analysis $\phi(t) \sim e^{v t}$. In this case, the above expression reduces to

$$
\Delta H=-\ln [f F(\mathbf{x})]
$$

where 


$$
F(\mathbf{x})=\int_{0}^{\infty} d t^{\prime} e^{-v t^{\prime}} g\left(\mathbf{x}, t^{\prime}+l^{2}\right)
$$

This expression is valid for $|\mathbf{x}| \ll v^{1 / 2} t$. For this entire range we see that the height deviation $\Delta h$ is independent of time. For extremely large distances from the forcing site $\left(|\mathbf{x}| \gg v^{1 / 2} t\right)$, the influence of the force is insufficient to induce a velocity, and the surface distortion is essentially zero.

We now concentrate on examining the form of the steady-state profile given by Eqs.(11) and (12). In fact there are two spatial regimes for this profile, separated by the length scale $R_{c}=v^{-1 / 2}$ which will emerge as a critical radius. We shall denote the regime $R_{c} \ll|\mathbf{x}| \ll$

$v^{1 / 2} t$ as region $\mathrm{A}$, and the regime $l \ll|\mathbf{x}| \ll R_{c}$ as region $\mathrm{B}$. It is important to stress that for $v t \gg 1$ and $v \ll 1$ (which is the case when $f \searrow f_{c}$ ), regions $\mathrm{A}$ and $\mathrm{B}$ are both large and well-separated. Region A is less interesting and simpler to analyse, so we shall focus on this first.

\section{A. $\Delta H$ in region $\mathbf{A}$}

In this region, $F(\mathbf{x})$ may be evaluated using a steepest-descents treatment. A straightforward calculation yields the result

$$
\Delta H(\mathbf{x})= \begin{cases}2|\mathbf{x}| / R_{c}+\mathrm{O}\left(\ln \left(|\mathbf{x}| / R_{c}\right)\right), & d<2 \\ 2|\mathbf{x}| / R_{c}+\mathrm{O}\left(\ln \left(|\mathbf{x}| / R_{c}\right)\right)+\mathrm{O}\left(\ln \left(R_{c} / l\right)\right), & d>2\end{cases}
$$

The form of the corrections is of interest. We see that for $d<2$, the leading term dominates for $|\mathbf{x}| \gg R_{c}$ as expected. This indicates that the overall height deviation within the critical radius is of order unity. For $d>2$ the microscopic length scale enters into the corrections and the condition for the dominance of the leading term is now $|\mathbf{x}| \gg R_{c} \ln \left(R_{c} / l\right)$. We are therefore given the hint that for $d>2$ the overall deviation of the height within the critical radius may be large (of order $\ln \left(R_{c} / l\right)$ ). To see this explicitly we now turn our attention to the form of $\Delta H$ for $|\mathbf{x}| \ll R_{c}$. 


\section{B. $\Delta H$ in region $\mathbf{B}$}

Although the integral determining $F(\mathbf{x})$ appears quite simple, the analysis in region B is rather complicated. We therefore refer the reader to Appendix A where this analysis is described in some detail. The end result for $\Delta H$ in region $\mathrm{B}$ takes the form

$$
\Delta H= \begin{cases}c(d) f|\mathbf{x}|^{2-d}+\mathrm{O}\left(\left(|\mathbf{x}| / R_{c}\right)^{2}\right), & d<2 \\ 2 f \ln (|\mathbf{x}| / l)+\mathrm{O}(f), & d=2 \\ (d-2) \ln (|\mathbf{x}| / l)+\mathrm{O}(1), & d>2\end{cases}
$$

where $c(d)=2 \Gamma(d / 2) /(2-d)$. [It is instructive for $d=2$ to rewrite the prefactor of the height deviation exclusively in terms of $R_{c}$ - making use of the relation $\left.2 f=1 / \ln \left(R_{c} / l\right)\right]$.

There are two main features of these results we wish to stress. First, there exists for all dimensions a critical radius $R_{c}$ within which the surface profile undergoes a distortion from the linear form shown in Eq.(13). The critical radius scales with $\delta f \equiv f-f_{c}$ as $R_{c} \sim(\delta f)^{-\nu}$ where $\nu(d)=\alpha(d) / 2$. In particular we note that for $2<d<4, \nu(d)=1 /(d-2)$; and for $d>4, \nu(d)=1 / 2$.

The second important point is that by calculating the overall height distortion within the critical radius, i.e. $\Delta H\left(R_{c}\right)$, we see that

$$
\Delta H\left(R_{c}\right) \sim \begin{cases}\mathrm{O}(1) & 0 \leq d \leq 2 \\ \ln \left(R_{c} / l\right) & d>2\end{cases}
$$

These results confirm the ideas propounded earlier, i.e. for $d \leq 2$ the distortion of the surface within the critical radius is negligible, whereas for $d>2$ the distortion is a macroscopic rupture which scales logarithmically with distance.

\section{APPLICATIONS TO KPZ WEAK/STRONG-COUPLING TRANSITION}

As we mentioned in the Introduction, there are two main characteristics of the weak/strong coupling transition for the noisy KPZ equation (which arise from a RG study in powers of $\epsilon=d-2$ ) for $d>2$. These are the vanishing of the roughness exponent, which 
implies a logarithmic roughness; and the existence of a correlation length $\xi \sim\left(g-g_{c}\right)^{-\tilde{\nu}}$, where $g$ is the dimensionless coupling constant, and $\tilde{\nu}=1 / \epsilon+O(\epsilon)^{3}$. There is a remarkable correspondence between these results, and those found in the previous section for the simple model of a deterministic KPZ interface under a local applied force. To reiterate these results, we found that for $d>2$, the applied force induces a logarithmic distortion of the surface within a critical radius which scales as $R_{c} \sim\left(f-f_{c}\right)^{-\nu}$ with $\nu=1 / \epsilon$ for $2<d<4$, and $\nu=1 / 2$ for $d>4$.

The natural question arises: are the critical properties of the noisy KPZ equation directly related to the simple problem discussed above? If so, then we have a much easier way to understand the weak/strong coupling phase transition, and also we may identify an upper critical dimension of 4 (for the unstable fixed point characterising the transition). A quantitative connection between the two sets of results is beyond our reach. However we shall make several points which certainly make the connection more plausible. Since we are interested in the weak/strong coupling transition, we henceforth restrict our attention to $d>2$.

The coupling constant $g$ in the noisy KPZ equation is proportional to the noise amplitude $D$ which we shall take to be our control parameter. For $D$ well below the critical value, the non-linearity in the KPZ equation is irrelevant. Thus, the surface behaves as an EdwardsWilkinson (EW) interface [10], which is smooth. As we increase the value of D, it becomes more and more likely for small regions in the interface to experience strong fluctuations due to rare events in the noise spectrum. If the smooth/rough transition is triggered by an inherent instability of the interface with respect to strong fluctuations, it is plausible to assume that such an instability is localized due to the local nature of rare events in the noise. Having established this, we may connect the two models by arguing that these strong local fluctuations resemble an effective force over long time scales. There are three essential steps in this argument which we discuss in turn.

First, we must argue that the fluctuations appear as a constant force over some temporal interval $\tau$. Clearly any fluctuations in the 'downward' direction (i.e. anti-parallel to the 
direction of positive $h$ ) will have no effect since they are completely suppressed by the nonlinearity. We therefore consider a noise path (or noise 'history') which tends on average to be positive over the interval $\tau$ within some region $\Lambda$ of size $l^{d}$. This path may be considered as a force of magnitude $f$ so long as it lies between the bounds $f \pm \sigma$. The fluctuations within these bounds are irrelevant. This fact may be established by considering the delta-function model Eq.(3) but allowing for the force to be a stochastic variable. By power counting one may show that the fluctuations of the force are irrelevant (about the constant force fixed point) for $d>1$. For the noise path to exceed $f+\sigma$ is extremely unlikely as the path is already a rare event even to have entered between these bounds. The path is much more likely to drop below $f-\sigma$. This is deemed as switching off the 'force'. The force will be switched on again when the noise path returns within the bounds. Thus the interval $\tau$ over which the force acts is a random variable.

Second, we must argue that this random switching off and on of the effective force is unimportant. We assume that the force has been able to create some disturbance in the interface over the region $\Lambda$, before it is switched off. How will the surface evolve? To answer this question one may simply solve the deterministic KPZ equation in the absence of any source, but with an initial condition corresponding to a localized disturbance. The result is that for any disturbance exceeding a critical height $h_{c} \sim \nu / \lambda$, the centre of the disturbance decays logarithmically slowly - it is essentially frozen. This effect is due to the strong influence of the non-linearity driving the disturbance upwards against the smoothing effect of the diffusion term. So during the periods of zero force, the disturbance in the region $\Lambda$ is frozen and on switching on the force, the evolution continues as if there were no interruption.

The third and final point is to argue that the effective force remains localized in the region $\Lambda$. This is in general not the case. The noise has short-range correlations in space and time and rare events are equally likely to occur anywhere in the surface. A simple resolution would be that once the rupture has been formed in $\Lambda$, then all other rare events in the vicinity may be rotationally averaged to give an effective force within $\Lambda$. A more subtle point is the following. Once the logarithmic distortion centered in $\Lambda$ has been established, it 
is essentially frozen during the periods of zero force. Therefore any rare event occurring in $\Lambda$ will continue the evolution of the distortion. On the other hand, if the critical height for freezing is not small, then most rare events will fail to seed such a distortion of the interface, since the disturbances due to rare events which are of a height less than $h_{c}$ will simply diffuse away. We therefore have a picture of extremely strong fluctuations being required to seed a distortion of height $>h_{c}$, with less strong fluctuations being required to sustain the evolution of the logarithmic rupture once it has been seeded.

If this rough physical picture is correct then the correspondence between the local force model considered here, and the noisy KPZ equation at the weak/strong-coupling transition is established. The surface morphology resulting from such a picture is that of a dilute system of macroscopic distortions, each with a logarithmic profile. This is in contradistinction to the more conventional view of weak uniform fluctuations giving rise to logarithmic roughness. Such a difference in morphology should be discernible from numerical simulations of models believed to lie in the KPZ universality class.

[A physically plausible connection between these two models may be drawn also in the directed polymer representation. The strong-coupling phase of the KPZ model corresponds to the low-temperature phase of the directed polymer, in which the polymer is stongly localized onto low-energy paths, which have characteristic transverse fluctuations. Conversely, the weak-coupling phase of KPZ corresponds to the high-temperature phase of the directed polymer in which the polymer is liberated from the low energy path and performs thermal wandering. Hence the transition between these two phases for the directed polymer may be viewed as the localization transition to the lowest energy path (at this temperature). In this case the polymer is essentially subjected to a 'columnar' defect, although the defect is not of constant energy, and also has transverse fluctuations. Using similar arguments to those presented above in the interface language, one can motivate the idea that i) the energy fluctuations on the column are irrelevant within some bounds, and ii) the transverse fluctuations of the column are thermally averaged such that the effective column is straight on some transverse scale of order $l^{d}$. We believe a more quantitative connection may be 
tractable in this directed polymer picture.]

\section{CONCLUSIONS}

We have presented a non-perturbative analysis of the deterministic KPZ equation under the influence of a local driving force $\mathrm{f}$, which for $d>2$ must exceed a critical value $f_{c}$ in order to induce a non-zero velocity in the surface. The central result is that for $d>2$ and for $f \searrow f_{c}$, the force creates a macroscopic distortion of the surface. This distortion has a logarithmic profile and exists within a critical radius of size $R_{c} \sim\left(f-f_{c}\right)^{-\nu}$ where $\nu(d)=1 /(d-2)$ for $2<d<4$ and $\nu(d)=1 / 2$ for $d>4$. In the penultimate section we offered some physical arguments as to why one might consider this simple model to underlie the weak/strong-coupling transition in the noisy KPZ model. The main characteristics of such a scenario are that i) the logarithmic roughness at the transition is due to a dilute system of logarithmic distortions, as opposed to a uniform sea of fluctuations with logarithmic variance, and ii) the critical radius $R_{c}$ is a physical realization of the correlation length found from the RG treatment. A more quantitative connection between these two models is presently beyond our reach, although one future possibility is to estimate the $d$-dependence of the weak/strong-coupling phase boundary based on the exact results obtained in this paper for the local force model, in conjunction with the statistical arguments presented above.

It would be useful to apply the non-perturbative methods presented here to the case of a random local force. Such an analysis would allow one to tighten some of the arguments given in section 5 . This problem is also of substantial interest in its own right as it may be mapped to a system of two directed polymers with random contact interactions [8] [11] [12] which has a wide range of applications.

The authors wish to thank M.A.Moore and L-H. Tang for illuminating discussions. TJN acknowledges financial support under SFB 341. 


\section{APPENDIX A:}

In this appendix we analyse the function $F(\mathbf{x})$ in region B, i.e. for $l \ll|\mathbf{x}| \ll R_{c}=v^{-1 / 2}$, in order to derive the relations shown in Eq.(14). The function $F(\mathbf{x})$ has the explicit form (cf. Eq.(12))

$$
F(\mathbf{x})=\int_{0}^{\infty} d t\left(t+l^{2}\right)^{-d / 2} \exp (-v t) \exp \left[-x^{2} /\left(t+l^{2}\right)\right]
$$

which may be re-expressed as

$$
F(\mathbf{x})=x^{2-d} e^{v l^{2}}\left[I_{1}(x)+I_{2}(x)\right]
$$

where

$$
I_{1}(x)=\int_{(l / x)^{2}}^{1} d t t^{-d / 2} e^{-a t} e^{-1 / t}
$$

and

$$
I_{2}(x)=\int_{1}^{\infty} d t t^{-d / 2} e^{-a t} e^{-1 / t}
$$

where we have defined $a \equiv v x^{2}=\left(x / R_{c}\right)^{2} \ll 1$ for notational convenience.

The first integral may be simply evaluated to leading order in $a$. We have

$$
I_{1}(x)=\int_{1}^{\infty} d u u^{d / 2-2} e^{-u}+\mathrm{O}(a)+\mathrm{O}\left(e^{-(x / l)^{2}}\right) .
$$

The leading terms of the second integral may be extracted as follows. Referring to Eq.(A4), we expand the last factor of the integrand as a power series to give

$$
I_{2}(x)=\sum_{n=0}^{\infty} \frac{(-1)^{n}}{n !} J_{n}(a)
$$

where

$$
J_{n}(a)=a^{n-1+d / 2} \int_{a}^{\infty} d t t^{-n-d / 2} e^{-t} .
$$

Integrating by parts yields the recursion relation 


$$
J_{n}(a)=\frac{e^{-a}}{(n-1+d / 2)}-\frac{a}{(n-1+d / 2)} J_{n-1}(a)
$$

For $d<2, J_{0}(a)$ has the expansion

$$
J_{0}(a)=\Gamma(1-d / 2) a^{d / 2-1}-(1-d / 2)^{-1}+\mathrm{O}(a)
$$

where $\Gamma(z)$ is the Gamma function [9]. This form of $J_{0}$ implies $J_{1}(a)=2 / d+\mathrm{O}\left(a^{d / 2}\right)$ and for $n>1, J_{n}=(n-1+d / 2)^{-1}+\mathrm{O}(a)$. Returning to Eq. (A6) we have

$$
I_{2}(x)=\Gamma(1-d / 2) a^{d / 2-1}+C+\mathrm{O}\left(a^{d / 2}\right)
$$

where

$$
C=\sum_{n=0}^{\infty} \frac{(-1)^{n}}{n !}(n-1+d / 2)^{-1}=(d / 2-1)^{-1}+\int_{0}^{1} d u u^{d / 2-2}\left[e^{-u}-1\right] .
$$

Combining Eqs.(A5) and (A10) then gives for $d<2$

$$
I_{1}+I_{2}=\Gamma(1-d / 2) a^{d / 2-1}-(1-d / 2)^{-1} \Gamma(d / 2)+\mathrm{O}\left(a^{d / 2}\right)
$$

From Table I we have for $d<2, v=[f \Gamma(1-d / 2)]^{2 /(2-d)}$, which in addition to Eqs.(A2) and (A12) gives

$$
f F(\mathbf{x})=1-f(1-d / 2)^{-1} \Gamma(d / 2)|\mathbf{x}|^{2-d}+\mathrm{O}\left(\left(|\mathbf{x}| / R_{c}\right)^{2}\right) .
$$

Combining the above equation with Eq.(11) then yields the first relation in Eq.(14).

A similar analysis suffices for higher dimensions, which we shall briefly sketch. For $d=2$ one finds

$$
I_{1}+I_{2}=-\ln (a)-2 \gamma+\mathrm{O}(a \ln (a))
$$

where $\gamma=0.57721 \ldots$ is Euler's constant. From Table I we have for $d=2, v=l^{-2} e^{-1 / f}$, which in addition to Eqs. (A2) and (A14) gives

$$
f F(\mathbf{x})=1-2 f \ln (|\mathbf{x}| / l)+O(f) .
$$

Combining the above equation with Eq.(11) then yields the second relation in Eq.(14). 
Finally for $d>2$ one finds

$$
I_{1}+I_{2}=\Gamma(d / 2-1)+\mathrm{O}\left(a^{\beta}\right)
$$

where $\beta=d / 2-1$ for $2<d<4$, and $\beta=1$ for $d>4$. From Table I we have for $d>2$, $f \sim f_{c}=(d / 2-1) l^{d-2}$, which in addition to Eqs.(A2) and (A16) gives

$$
f F(\mathbf{x})=\Gamma(d / 2)(l /|\mathbf{x}|)^{d-2}+\mathrm{O}\left(\left(|\mathbf{x}| / R_{c}\right)^{2 \beta}(l /|\mathbf{x}|)^{d-2}\right)
$$

Combining the above equation with Eq.(11) then yields the third relation in Eq.(14). 
Table I

\begin{tabular}{|c||c|c|c|c|c|}
\hline \multirow{2}{*}{$d$} & \multirow{2}{*}{$f_{c}$} & \multicolumn{3}{|c|}{$H(t)$} & \multirow{2}{*}{$v(f)$} \\
\cline { 3 - 5 } & & $f<f_{c}$ & $f=f_{c}$ & $f>f_{c}$ & \\
\hline \hline $0 \leq d<2$ & 0 & $-\frac{(2-d)}{2} \ln \left(f^{2 /(2-d)} t\right)$ & 0 & $v t$ & $c_{1}(d) f^{2 /(2-d)}$ \\
\hline 2 & 0 & $-\ln \left(\ln \left(t / l^{2}\right)\right)$ & 0 & $v t$ & $\frac{1}{l^{2}} \exp (-1 / f)$ \\
\hline $2<d<4$ & $\frac{(d-2)}{2} l^{d-2}$ & $-\ln \left(1-\frac{f}{f_{c}}\right)$ & $\frac{(d-2)}{2} \ln \left(t / l^{2}\right)$ & $v t$ & $\frac{c_{2}(d)}{l^{2}}\left(\delta f / f_{c}\right)^{2 /(d-2)}$ \\
\hline 4 & $l^{2}$ & $-\ln \left(1-\frac{f}{f_{c}}\right)$ & $\ln \left[\frac{t / l^{2}}{\ln \left(t / l^{2}\right)}\right]$ & $v t$ & $\frac{1}{l^{2}} \frac{\left(\delta f / f_{c}\right)}{\ln \left(f_{c} / \delta f\right)}$ \\
\hline$d>4$ & $\frac{(d-2)}{2} l^{d-2}$ & $-\ln \left(1-\frac{f}{f_{c}}\right)$ & $\ln \left(t / l^{2}\right)$ & $v t$ & $\frac{1}{l^{2}} \frac{(d-4)}{2}\left(\delta f / f_{c}\right)$ \\
\hline
\end{tabular}

\section{Caption for Table I}

Asymptotic behaviour of $H(t)$ - the interface height at the forcing site - as a function of dimension $d$ and applied force $f$. The symbol $\delta f$ is the excess force: $\delta f=f-f_{c}$. The constants $c_{1}$ and $c_{2}$ have the form $c_{1}(d)=[\Gamma(1-d / 2)]^{2 /(2-d)}$ and $c_{2}(d)=[\Gamma(2-d / 2)]^{2 /(2-d)}$. 


\section{REFERENCES}

* address from 1st January 1996: Department of Physics, University of Manchester, Manchester, M13 9PL, U.K.

[1] KARDAR M., PARISI G. and ZHANG Y-C., Phys. Rev. Lett. 56 (1986) 889.

[2] KRUG J. and SPOHN H., in Solids Far From Equilibrium: Growth, Morphology and Defects, C. Godreche Ed. (Cambridge, Cambridge University Press 1991).

[3] HALPIN-HEALY T. and ZHANG Y-C., Phys. Rep. 254 (1995) 215.

[4] MOORE M. A., BLum T., DOHERTY J. P. and MARSILI M., Phys. Rev. Lett. 74 (1995) 4257.

[5] FREY E. and TÄUBER U. C., Phys. Rev. E 50 (1994) 1024.

[6] NATtermann T. and TANG L-H., Phys. Rev. B 45 (1992) 7156.

[7] LANDAU L. D. and LIFSHITZ E. M., Quantum Mechanics (Pergamon Press, London 1958).

[8] KALLABIS H. and LÄSSIG M., Phys. Rev. Lett. 75 (1995) 1578.

[9] Handbook of Mathematical Functions 10th edition, M. Abramowitz and I. A. Stegun Eds. (New York, Dover 1972).

[10] EDWARDS S. F. and WILKINSON D. R., Proc. R. Soc. London Ser. A 381 (1982) 17.

[11] LIPOWSKY R., Europhys. Lett. 15 (1991) 703. In this reference $d=4$ was found to play a role similar to that noted in the present analysis.

[12] DERRIDA B., HAKIM V. and VANnimenus J., J. Stat. Phys. 66 (1992) 1189. BHATTACHARJEE S. M. and MUKHERJI S., Phys. Rev. Lett. 70 (1993) 49. 